$$
\begin{aligned}
& 110 \\
& \therefore \cdot 0= \\
& 424919
\end{aligned}
$$

\title{
Overview of Sensitivity Analysis and Shape Optimization for Complex Aerodynamic Configurations
}

\author{
James C. Newman, III \\ Mississippi State University \\ Mississippi State, MS 39762
}

Richard W. Barnwell

Virginia Polytechnit Institute and State Unive i. ity

clo Virginia Consortium if Engineering \&

Science Universities, Hampton, VA 23666
Arthur C. Taylor, III

Old Dominion University Norfolk, VA 23529

Perry A. Newman
NASA Langley Research Center

Hampton, VA 23681

June 1998

Submitted to

AIAA Journal of Aircraft,

for Publication in Special MDA \& O Issue 



\begin{abstract}
This paper presents a brief overview of some of the more recent advances in steady aerodynamic shape-design sensitivity analysis and optimization, based on advanced computational fluid dynamics. The focus here is on those methods particularly wellsuited to the study of geometrically complex configurations and their potentially complex associated flow physics. When nonlinear state equations are considered in the optimization process, difficulties are found in the application of sensitivity analysis. Some techniques for circumventing such difficulties are currently being explored and are included here. Attention is directed to methods that utilize automatic differentiation to obtain aerodynamic sensitivity derivatives for both complex configurations and complex flow physics. Various examples of shape-design sensitivity analysis for unstructured-grid computational fluid dynamics algorithms are demonstrated for different formulations of the sensitivity equations. Finally, the use of advanced, unstructured-grid computational fluid dynamics in multidisciplinary analyses and multidisciplinary sensitivity analyses within future optimization processes is recommended and encouraged.
\end{abstract}

\title{
Background Remarks
}

Numerical optimization procedures are based on mathematical techniques for finding the extremum of an objective function subject to various constraints. When the costs associated with evaluating the objective function and constraints are excessive (as in the case of nonlinear aerodynamic analyses), zeroth-order methods are usually prohibitively expensive and, thus, gradient-based design optimization procedures are frequently adopted. These procedures require the gradients of the objective function and constraints (dependent variables) with respect to the shape-design (independent) variables. These gradients, commonly referred to as sensitivity derivatives, provide a mechanism for changing the design variables to improve the objective function without violating the given constraints. Sensitivity derivatives may be evaluated by finite differencing; however, this approach is not only computationally expensive, but unless carefully monitored it can produce inaccurate gradient approximations. The preferable approach is to obtain the sensitivity derivatives analytically. The analytic evaluation of sensitivity derivatives requires an additional level of simulation referred to as sensitivity analysis.

For aerodynamic optimization, the state equation is a system of nonlinear partial differential equations (PDE) expressing the conservation of mass, momentum, and energy. Differentiation of the system of PDE (i.e., sensitivity analysis) can be performed at one of two levels. In the first method, termed the continuous or variational approach, the PDE are differentiated prior to discretization, either directly or by introducing Lagrange multipliers which are defined by a set of continuous linear equations adjoint to the governing PDE. Subsequently, these directly differentiated or adjoint equations are discretized and solved. In the second method, termed the discrete approach, the PDE are 
differentiated after discretization. The discrete approach may also be cast in either a direct or an adjoint formulation, and the reader should refe to Hou et al. ${ }^{1}$ for a comprehensive presentation of both discrete formulations. For more detailed recent discussions of the continuous approaches to aerodynamic design optimization, the interested reader is directed, for example, to Refs. 2-6 for the adjoint formulation and to Refs. 7 and 8 for the direct formulation.

The task of constructing exactly or analytically all of the required linearizations and derivatives by hand for either the discrete or continuous approach and then building the software for evaluating these terms can be extremely tedious. This problem is compounded by the inclusion of even the most elementary turbulence model (for viscous flow) or the use of a sophisticated grid generation package for adapting (or regenerating) the computational mesh to the latest design. A promising possible solution to this problem, however, has been found in the use of a technique known as automatic differentiation. Application of this technique to an existing source code, that evaluates output functions, automatically generates another source code that evaluates both output functions and derivatives of those functions with respect to specified code input or internal parameters. One such precompiler software tool, called ADIFOR (Automatic DIfferentiation of FORtran, Bischof et al. ${ }^{9-11}$ ), has been developed and utilized with much success to obtain complex derivatives, from advariced computational fluid dynamics (CFD) and grid generation codes, for use within aerodynamic design optimization procedures ${ }^{12-18}$. The use of ADIFOR produces code that, when executed, evaluates these derivatives of the output functions via a discrete-dire t approach, referred to as forwardmode automatic differentiation. More recently, autsmatic differentiation software has emerged that enables the derivatives to be evaluated with a discrete-adjoint approach ${ }^{19,20}$. This type of automatic differentiation is known as reverse-mode ${ }^{21}$.

The selection of an appropriate sensitivity analysis formulation (direct or adjoint) depends on the particular design problem being studied, regardless of whether or not the linearizations were constructed by hand differentiation or by automatic differentiation. The computational work for the direct approach scales with the number of design variables; that is, a linear system of equations must be solved for the derivatives with respect to each design variable. For adjoint formulations, the number of linear systems which must be solved scales with the number of augmented output functions. Generally there is one system for the adjoint corresponding to the objective function and one for each augmented contsraint that is a function of the state vector. In multidisciplinary design optimizations that involve coupled discipline input and output, adjoints for additional augmented output functions may be require $d$.

The early aerodynamic shape optimization work has been reviewed by Labrujere and Slooff ${ }^{22}$. A concise review of the use of sensitivity analysis in aerodynamic shape optimization has been previously reported by Taylor et al. ${ }^{23}$. An AGARD-von Karman Institute special course ${ }^{24}$ in 1994 was devoted to optimum design methods in aerodynamics. The reader is urged to see these sources for earlier research not cited in the current overview. In a later von Karman Institute lesture series ${ }^{25}$, optimization methods 


\title{
Overview of Sensitivity Analysis and Shape Optimization for Complex Aerodynamic Configurations
}

\author{
James C. Newman, III ${ }^{1}$ \\ Mississippi State University \\ Mississippi State, MS 39762 \\ Arthur C. Taylor, III $^{2}$ \\ Old Dominion University \\ Norfolk, VA 23529 \\ Richard W. Barnwell ${ }^{3}$ \\ Virginia Polytechnic Institute and State \\ University \\ clo Virginia Consortium of Engineering \& \\ Perry A. Newman ${ }^{4}$ \\ NASA Langley Research Center \\ Hampton, VA 23681 \\ Science Universities, Hampton, VA 23666 \\ Gene J.-W. Hou ${ }^{5}$ \\ Old Dominion University \\ Norfolk, VA 23529 \\ revised , 6-19-98

\footnotetext{
1 Assistant Professor, Department of Aerospace Engineering, Member AIAA.

2 Associate Professor, Department of Mechanical Engineering, Member AIAA.

3 Professor, Aerospace and Ocean Engineering Department \& Engineering Science and Mechanics Department, Fellow AIAA.

4 Senior Research Scientist, Multidisciplinary Optimization Branch, Mail Stop-159.

5 Professor, Department of Mechanical Engineering, Member AIAA.
} 

for flow control and aerodynamic shape design are reviewed and some more recent applications are presented. A recent paper by Jameson ${ }^{26}$ delineates the evolution of computational fluid dynamics as a design tool.

The current paper is a brief overview of gradient-based shape optimization research that focuses on those methods and techniques that are suitable for application to complex aerodynamic configurations and their associated flow physics. Undoubtedly, some recent works have been overlooked and not all works of the cited research groups have been included in this overview. This field is currently very active and expanding; a number of commercial aerospace design activity results are proprietary and hence are not available now in the open literature.

\section{Overview of Recent Advances in Aerodynamic Shape Optimization}

Using advanced CFD in aerodynamic shape optimization presents some challenges and difficulties. This overview discusses some of these issues, then reviews some structuredgrid and unstructured-grid CFD applications.

\section{Challenges and Difficulties for Advanced CFD}

In the mid 1970s Hicks et al. ${ }^{27-29}$ began exploring the use of numerical optimization techniques for the design of aircraft components. These early studies focused primarily on airfoil and wing design using the lower fidelity nonlinear transonic small disturbance equation or the full potential equation for the analyses and using finite-difference calculations for gradient information. These analyses were limited in their ability to accurately predict nonlinear phenomena. Also during this time, uses of optimal control techniques were being explored by Pironneau et al. ${ }^{30-32}$, for example, in incompressible viscous flows, to obtain analytical gradients. In the early 1980s Angrand ${ }^{33}$ applied these optimal control techniques to compressible potential flow equations for 2-D airfoils. These techniques and the early French studies are discussed by Pironneau ${ }^{34}$. Independently, Jameson ${ }^{2}$ advocated and used control theory techniques in aerodynamic design via CFD.

By the mid 1980s computational resources were available that permitted aerodynamic flow simulations using the higher fidelity compressible Euler and Navier-Stokes equations; these simulations were for isolated components and moderately complex configurations. Then Sobieski ${ }^{35}$ challenged the aerodynamics community to extend its CFD algorithms to include the shape sensitivity analysis of the geometry. This plea ignited studies at NASA Langley aimed at developing methods that would allow for the use of nonlinear aerodynamics in shape optimization. However, to become useful design tools, these aerodynamic optimization procedures require the capability to analyze and design complex configurations of practical interest. As recently noted by Elliot and 
Peraire ${ }^{36}$, this capability may provide "the step that determines the economic viability of the vehicle."

With improvements in computer speed and memory as well as advances in computer architectures, numerous aerodynamic design optimization procedures, 3 , 12,37-56 have emerged which directly couple the fields of computational fluid dynamics, sensitivity analysis, and numerical optimization. These procedures have enormous potential as design tools and are therefore receiving considerable attention in the aerospace, automotive, and biomedical research communities (among others). Difficulties, however, associated with the in-core memory needed for the analytic evaluation of discrete sensitivity derivatives, and with the considerable computational (CPU) time required to perform the subsequent optimization, are currently being addressed by various researchers. Dramatic in-core memory reductions have been accomplished by Newman et al. ${ }^{41,57,58}$ via the use of an incremental iterative solution of the sensitivity equation ${ }^{43}$ where memory-efficient methods were used to construct Jacobian matrix-vector products (as in Refs. 59 and 60, for example). The other limiting factor governing the acceptance of these shape optimization procedures is the large CPU times incurred when nonlinear fluid models are considered. Reductions of the excessive CPU run times required to perform the design optimization are being explored through the use of simultaneous (aerodynamic) analysis and design optimization (SAND) ${ }^{48,51,61-63}$, one-shot ${ }^{49}$, and pseudo-time ${ }^{64,65}$ methods, and parallel computing architectures ${ }^{12,66}$.

As recently noted by Reuther et al..$^{67}$ "While flow analysis has matured to the extent that Navier-Stokes calculations are routinely carried out over very complex configurations, direct CFD based design is only just beginning to be used in the treatment of moderately complex three-dimensional configurations." This shortcoming is primarily due to the difficulty, perhaps even impossibility, o generating a single structured grid about such a configuration. To handle a typical complex geometry of practical interest, some sort of domain decomposition must be incorporated into the design code. For structured-grid solvers, decomposition methods include multiblocked ${ }^{68,69}$, zonally patched $^{70,71}$, and overlapped ${ }^{72,73}$ (sometimes referred to as Chimera ${ }^{74}$ ) grid algorithms. However, as the geometric flexibility of the method increases, so does the complexity of the underlying algorithm. Since the use of sensitivity analysis to evaluate the needed gradients for a numerical optimizer is still evolving, imited work has been done toward extending structured-grid sensitivity algorithms to include these domain-decomposition methods. Unstructured-grid schemes provide an alterr ative to resorting to structured-grid domain-decomposition methods for coping with conplex configurations. Since triangles and tetrahedra are the simplest geometric shap ss possessing area and volume, respectively, they are capable of resolving irregularly shaped domains more easily and with greater efficiency. Another attribute of unstrictured grids is that they may be adapted and locally enriched where needed without affecting other regions of the mesh; thus, they may provide better control over mesh-dependent discretization errors. Examples of mesh refinement techniques for aerodyramic simulations may be found in Refs. 75 and 76. The state of the art for Euler and Navier-Stokes unstructured-grid 
solvers has been recently reviewed by Venkatakrishnan ${ }^{77}$, and he discusses some future research directions. The reader should consult this source for the status of unstructuredgrid CFD.

\section{Structured-Grid Based CFD Applications}

Research concerning the incorporation of the structured-grid domain-decomposition techniques has concentrated mostly on the use of multiblocked grids. To this end, Reuther et al. ${ }^{67}$ have extended the continuous or control theory approach of Jameson ${ }^{2}$ to produce a multiblock-multigrid, adjoint solver. In Ref. 67, this approach has been applied to the inviscid wing redesign of a transonic business jet. In Ref. 66 , this algorithm was subsequently extended to include parallel computations and was applied again to the transonic business jet configuration as well as to a supersonic transport configuration. Jameson et al. ${ }^{78}$ also extended this algorithm to viscous aerodynamic design; however, the viscosity and heat conduction coefficients are considered independent of the flow and are therefore not differentiated. Eleshaky and Baysal ${ }^{79}$ developed a multiblock discrete adjoint solver that was applied to a simple axisymmetric nozzle near a flat plate. This approach was later utilized by Lacasse and Baysal ${ }^{80}$ for the viscous (laminar) design of two-dimensional, multi-element airfoil components.

For other domain-decomposition methods (zonal and overlapped grids), Taylor ${ }^{13,14}$ has differentiated the advanced flow-analysis code CFL3D ${ }^{81}$ to perform the discrete sensitivity analysis. This research utilized the automatic differentiation software tool $\mathrm{ADIFOR}^{9-11}$ and solved the equations in incremental iterative form ${ }^{43,82}$. Oloso and Taylor $^{12}$ have reported impressive CPU time savings through the use of parallel computations of the sensitivity derivatives. In addition, Sherman et al. ${ }^{83}$, utilizing the design code developed in Ref. 43, successfully combined the automatic and hand differentiations, in incremental iterative forms, to produce a hybrid scheme. Several strategies for computing second-order aerodynamic sensitivity derivatives (Hessian matrix) using various combinations of the discrete-direct and discrete-adjoint formulations were investigated; both laminar and turbulent airfoil flows were considered. The turbulence model used was that of Baldwin and Lomax ${ }^{84}$. Subsequently, this code was applied by Hou et al. ${ }^{85}$ to transonic, turbulent airfoil design optimization.

Automatic differentiation has been used by Green et al. ${ }^{15}$ to differentiate the threedimensional thin-layer Navier-Stokes solver TLNS3D ${ }^{86}$ with respect to both algorithm and viscous modeling parameters. Specifically, the Clauser and the Van Driest constants, as they appear in the Baldwin-Lomax algebraic turbulence model, were used as the independent-viscous modeling parameters. The dependent variables, or output functions, were the aerodynamic performance parameters of lift, drag, and pitching moment coefficients. The impetus of that work was to demonstrate the feasibility of obtaining accurate, complex sensitivity derivatives from state-of-the-art CFD modeling codes. 


\section{Unstructured-Grid Based CFD Applications}

The current effort to apply unstructured-grid CFD approaches in higher-fidelity, compressible aerodynamic design optimization began early in the 1990s. Beux and Dervieux ${ }^{4}$ demonstrated first-order spatially accurate sensitivity analysis and optimization of a two-dimensional nozzle. They used a continuous adjoint method to derive the optimality conditions but reverted to a discrete approach for computer implementation. Later ${ }^{87}$, they studied the impact of second-order spatial accuracy for the state equations while computing the sensitivity derivatives with divided differences. Orozco and Ghattas ${ }^{51}$ proposed an infeasible path method, a SAND approach, where a Galerkin finite-element discretization of the nonlinear potential flow equation was performed. In Ref. 51, target-pressure distributions were matched on a subsonic airfoil, using what was referred to as the coordinate basis infeasible path method. Ghattas and Bark $^{88}$ have recently developed a reduced Hessian sequential quadratic programming method that, once again, is a SAND approach. This method is used in Ref. 88 for optimal flow control, simulated by steady, incompressible, laminar Navier-Stokes equations. A two-dimensional cylinder and a three-dimensional sphere are used as demonstrative examples. In this work, fluid suction and injection at the boundaries are used to affect flow control; the rate at which energy is dissipated in the fluid is the objective function.

Newman et al. ${ }^{89}$ developed a two-dimensional, and later a three-dimensional ${ }^{57}$, secondorder spatially accurate discrete sensitivity analysi; approach that has been used to perform the inviscid design optimization of airfoils ard transport wings in transonic flow. Included in Refs. 57 and 89 are optimization results from a limited study of the influence of spatial accuracy for both the state equations and sensitivity analyses. More recently, Newman ${ }^{41}$ and Newman et al. ${ }^{58}$ have presented the shape sensitivity analysis and design optimization of a subsonic, high angle-of-attack, multi-element airfoil and of a full Boeing 747-200 aircraft. In the work of Refs. 57, 58, and 89, the unstructured grid sensitivities with respect to the geometric design variables are evaluated by differentiating the grid adaptation and surface parameterization routines with ADIFOR. Once the shape sensitivity analysis code has been developed, the only modules that change from one configuration to another are these surface parameterization routines. Hence, utilizing ADIFOR for this purpose provided an efficient and accurate means of studying various geometries.

Elliot and Peraire ${ }^{36}$ also developed an inviscid, unstructured-grid, discrete-adjoint sensitivity analysis approach that was used to matclı target pressure distributions for a two-element airfoil and a wing-body configuration. Subsequently, they ${ }^{42}$ applied their algorithm to perform the inverse pressure design of : wing for a transonic business jet. More recently, this group of researchers has extended their two-dimensional algorithm to include viscous (laminar) effects ${ }^{90}$ and has demons rated its capability in airfoil drag reduction optimization with lift constraints. In the work of Refs. 36, 42, and 90, the grid sensitivity terms are computed analytically. 
In a von Karman Institute lecture $\operatorname{series}^{25}$, Giles briefly discusses the use of unstructured grids in the aerodynamic design of complex geometries and reviews the various means by which the adjoint equations may be formulated. Giles presents two examples illustrating the use of unstructured grids in aerodynamic design. The first example deals with a turbomachinery design by his student, Shrinivas ${ }^{91}$. They redesigned the outlet guide vanes in the bypass duct of a turbofan engine to reduce the interference between the outlet guide vanes and the support pylon. The shape-design variables selected allow the camber of the outlet guide vanes to be varied in both the axial and the spanwise directions. The second example presented was that of Ref. 42, discussed above.

An equally impressive use of unstructured-grid approaches for the design of geometrically complex devices has been performed by Burgreen et al. ${ }^{92,93}$, who used CFDbased design optimization methods to improve the thrombogenic performance of an axial flow blood pump. This research of Burgreen et al. represents the expansion of traditional aerodynamic design optimization procedures into the biomedical field to aid in artificial heart design.

Anderson and Venkatakrishnan ${ }^{5}$ have developed an unstructured-grid approach to sensitivity analysis that actually utilizes a continuous adjoint approach for computer implementation as well as for the derivatives. Moreover, in Ref. 5 limitations of the continuous adjoint approach are discussed, and a hybrid continuous-discrete approach, which addresses some of these deficiencies, is developed. More recently, Anderson and Bonhaus $^{94}$ have extended the work of Ref. 5 to a fully discrete-adjoint approach and included turbulent Navier-Stokes design capabilities. The one-equation turbulence model of Spalart-Allmaras ${ }^{95}$ was consistently differentiated by hand as reported in Ref. 94, where the ability to match target pressure distributions was also demonstrated.

An example of reverse mode automatic differentiation applied to an unstructured grid algorithm has been reported by Mohammadi ${ }^{19}$, who used a discrete-adjoint approach to demonstrate two-dimensional airfoil drag reduction optimization for both inviscid and turbulent Navier-Stokes equations. The turbulence model used was a two-equation $\mathrm{k}-\varepsilon$ model with wall-laws. Mohammadi also demonstrates three-dimensional, inviscid, swept-wing drag reduction optimization with approximately 3,000 design variables.

\section{Sample of Unstructured-Grid Based CFD Sensitivity Analysis Results for Complex Geometries}

This section discusses shape-sensitivity analyses for several complex configurations of practical interest. Sample results from two different formulations for sensitivity analysis applied to unstructured-grid CFD algorithms are presented. These samples illustrate that sensitivity analysis methods of the type discussed in this overview are capable of evaluating flow sensitivities in the presence of complex physics and multi-component interactions that occur in geometrically complex configurations of practical interest. Details concerning the use of these sensitivity-analysis results for design optimization may be found in the cited literature. 


\section{Two-Dimensional Examples}

The first example is an application of discrete-direct sensitivity analysis for a fourelement, high-lift airfoil system. This system is composed of a main airfoil that carries most of the load, a leading-edge slat that aligns the flow onto the main airfoil, a main flap that provides camber for the high-lift system, and a vane that controls the flow off the main airfoil and onto the main flap. The unstructured Euler mesh for this geometrically complex configuration is shown in Fig. 1(a). In the design study of Ref. 58, the vane was parameterized with Bezier curves $^{96}$ and subsequently optimized for the maximum inviscid lift coefficient. The grid sensitivity terms were computed via ADIFOR-differentiated routines and verified by comparison with finite-difference values. Figure 1(b) depicts the grid sensitivity of the $y$-coordinates of the interior mesh with respect to a design variable (Bezier control point) on the lower surface of the vane. Contours of nondimensional pressure in the flow field about this high-lift system are shown in Fig. 1(c) for low speed flow at about $16 \mathrm{deg}$ incidence. As a qualitative illustration of the influence of shape changes on the flow field solution, Fig. 1(d) depicts the contours of the nondimensional pressure sensitivity with respect to this lower-surface vane design variable. It can be seen that surface pressures in the leading-edge region of the main flap are affected by this vane design variable. Such state-variable sensitivity derivatives are used in evaluating objective function and constraint gradients.

An example of a continuous-adjoint approach to sensitivity analysis has been given by Anderson and Venkatakrishnan ${ }^{5}$. In that example, the aft airfoil in a two-element airfoil configuration was redesigned to obtain a desired shockless pressure distribution on the front element. The unstructured Euler grid used in that study is shown in Fig. 2(a). Contours of nondimensional pressure in the initial flow field at a Mach number of 0.6 and at zero incidence are illustrated in Fig. 2(b). The region where these contours are very closely spaced and essentially parallel represents a shock wave standing between the two elements. Contours for the costate-variable (adjoint) component corresponding to streamwise momentum are shown in Fig. 2(c). This costate-vector component may be viewed as a component in a fictitious linearized time reversed flow and appears to exhibit a "shock-like" structure (Fig. 2(c)), located between the two elements at the (front) sonic line of the real flow (Fig 2(b)). This costate cornponent also exhibits "wake-like" structures emerging from the leading edges of both elements. The costate-variable components, such as that shown in Fig. 2(c), are used to evaluate the gradient of the objective function. Additional details have been noted in Ref. 5 .

\section{Three-Dimensional Example}

An example of shape-sensitivity analysis using the discrete-direct approach for a complete aircraft configuration was presented in Ref. 58. The unstructured Euler surface mesh for this four-engine configuration is shown in Iig. 3(a). This grid was constructed from the measured coordinates of a Boeing 747-200 rodel tested in the NASA Ames 11foot Transonic Pressure Tunnel (Test AR0502). Contururs of nondimensional pressure on 
the "upper" surfaces of this configuration are shown in Fig. 3(b) for transonic flow at a Mach number of 0.84 and at 2.73 deg incidence. A shock wave on the wing appears as the closely spaced contours. A qualitative illustration of the influence of shape changes on the flow field solution is shown in Fig. 3(c), which depicts contours of the nondimensional pressure sensitivity derivative with respect to a winglet-type design variable. That is, this design variable varies the dihedral linearly over the outermost stations of the wing. Note that, in addition to causing the localized flow-field disturbance in the region where the design variable changes the geometry, this geometry change also propagates a disturbance along the entire wing upper-surface shock wave.

\section{Incorporating Advanced CFD into Multidisciplinary Optimization (MDO)}

Traditional multidisciplinary design is a process that has been practiced by industry for many years. At the conceptual level of design, the discipline analyses are of a low fidelity, and multidisciplinary trade-offs to accomplish a design improvement can be understood and, in many cases, made without formal sensitivity analysis nor optimization. Decisions at the conceptual level, however, are based upon the low fidelity information.

At the next, or preliminary, level of design, the disciplinary analyses are of a higher fidelity and generally require a greater number of design variables. As the number of design variables grows, the multidisciplinary trade-offs or coordination to accomplish a design improvement are not at all obvious. If the required multidisciplinary coordination is even done, more often than not it is done at another level, after the sequential disciplinary analyses are cycled, and independent of the individual discipline expertise. To obtain multidisciplinary coordination, the sequential cycle must be repeated. These designs, and data for these designs, are produced sequentially at the discipline level, then transferred (thrown over the fence) to another organizational level or discipline.

It is noted in Ref. 97 that, following this traditional design procedure, "as the design process goes forward designers gain knowledge but lose freedom to act on that knowledge." This self-limiting procedure drives the need to coordinate the multidisciplinary process at the discipline level and to introduce higher fidelity computational models earlier in the design cycle where perhaps more revolutionary, as opposed to evolutionary, design improvements can be considered. Recommendations on how to reduce the cycle time associated with the design and development of aircraft have been proposed by Grose ${ }^{98}$ and by Jameson ${ }^{99}$. These papers propose a re-engineering of the design process which includes incorporation of more advanced CFD models; Jameson presents a case study conducted on the McDonnell Douglas MDXX.

Formal coordination of the multidisciplinary process at all levels can be considered as the theory of MDO, as opposed to the traditional practice of MDO described previously. The theory of MDO is an emerging field that attempts to use numerical optimization techniques to develop improved and efficient designs for complex, interacting, engineering 
systems. Detailed surveys of research being conducted in this area have been compiled by Sobieski ${ }^{100,101}$ and by Sobieski and Haftka ${ }^{102}$, and once again, the reader is directed to these sources. Numerous techniques have been proposed to accomplish the required coordination between the disciplines; in an attempt to obtain a perspective on the various methods being explored, Cramer et al. ${ }^{103}$, Balling and Sobieski ${ }^{104}$, and Newman et al ${ }^{82}$ have developed classifications of these techniques. These classifications are based on the problem decomposition and interaction of the ciscipline analyses, the sensitivity information, and the numerical optimizer. Examples of the first two of these three interaction elements, namely coupled discipline analyses and coupled sensitivity analyses, are given in the following two paragraphs. Much of the NASA Langley sponsored aerodynamic sensitivity analysis work for advanced CFD codes (some already cited herein) has been developed for ultimate use in MDO; this work has been reviewed, chronologically, in Refs. 105, 23, 106, and 82.

Engineering systems of practical interest are usually characterized by interactions between various disciplines. In some cases, simplifyng assumptions may be made that decouple these disciplines with reasonable accuracy. In other situations, the interactions themselves may produce changes in the system's response that are of the same order of magnitude as those of the decoupled discipline analyses. The design engineer must consider such disciplines simultaneously in order to evaluate the level of interaction that exists between the physical models being considered and, within the design process, must evaluate the sensitivity of system performance due to this interaction. The need for multidisciplinary analysis may be recognized by neglecting the structures discipline in the analysis of an aircraft wing in transonic flow. Large, elastic deformations may be present, and the predicted lift coefficients of aerodynamic-cnly versus aero-structural analysis have been found to differ significantly. This discrepancy is illustrated in Fig. 4(a), which depicts the convergence histories of rigid-wing aerodynamic analysis, aero-structural analysis, and aero-structural analysis trimmed to the rigid wing lift using the flow angle of attack. Considerable elastic deflection at transonic flow conditions for the untrimmed aero-structural analysis is shown in Fig. 4(b). The aerodynamic CFD mesh is much denser than the underlying structural FEM mesh; ho vever, both are relatively coarse by current disciplinary analysis practice. The reader nay refer to Ref. 41 or 107 for additional information about these analyses.

An illustration of the need for multidisciplinary sensitivity analysis in the design process has been presented by Arslan and Carls on ${ }^{108}$. These researchers obtained multidisciplinary sensitivity analysis results using, the transonic, small-disturbance potential equation for the fluid model and an equivalent flat plate model for the wing structure. The use of this simplified, nonlinear, fluil model allowed computations and sensitivities to be evaluated in the transonic regime. The resulting, ill-conditioned, global sensitivity equations ${ }^{109}$ were reformulated and solved by the incremental iterative technique mentioned previously ${ }^{43}$. Reference 108 demonstrated that the sensitivity information produced by an aerodynamic-only calculation had different magnitudes, and in some cases different signs, from those obtained $w$ th the coupled sensitivity analysis. 
Similar findings have been reported by Barthelemy and Bergen ${ }^{110}$ and by Newman et al. ${ }^{111}$.

\section{Concluding Remarks}

The trend toward increasing the aerodynamic complexity of aircraft configurations to which sensitivity analysis and shape optimization technology are applied will continue and probably accelerate. This trend is driven by the obvious benefits of increased aerodynamic performance and, additionally, by the benefits of synergistically improving performance in other aircraft disciplines such as structures and noise abatement. The difficulties peculiar to the application of sensitivity analysis and shape optimization within the discipline of aerodynamics, relate primarily to the need for concentrated grid density in high-gradient regions of flow. Unstructured-grid technology provides the most efficient means for fitting complex irregular shapes, refining grid where necessary, and accommodating gridding restraints from other disciplines such as structures.

\section{Acknowledgments}

The research conducted by the first, second, third, and fifth authors was partially supported by the NASA Graduate Student Research Program under grant NGT-51247 and by grant NAG-1-1265 from the Multidisciplinary Optimization Branch at NASA Langley Research Center. The authors would like to express their gratitude to Dr. W. K. Anderson for permitting the use of figures from Ref. 5 and for his many useful comments.

\section{References}

1. Hou, G. J.-W., Taylor III, A. C., and Korivi, V. M., "Discrete Shape Sensitivity Equations for Aerodynamic Problems," International Journal of Numerical Methods in Engineering, Vol. 37, 1994, pp. 2251-2266; also AIAA Paper 91-2259, June 1991.

2. Jameson, A., "Aerodynamic Design via Control Theory," Journal of Scientific Computing, Vol. 3, 1988, pp. 233-260; also ICASE Report No. 88-64 (NASA CR 181749), Nov. 1988.

3. Reuther, J. J., "Aerodynamic Shape Optimization Using Control Theory," Ph.D. Dissertation, University of California at Davis, May 1996.

4. Beux, F., and Dervieux, A., "Exact-Gradient Shape Optimization of a 2D Euler Flow," Finite Elements in Analysis and Design, Vol. 12, 1992, pp. 281-302.

5. Anderson, W. K., and Venkatakrishnan, V., "Aerodynamic Design Optimization on Unstructured Grids with a Continuous Adjoint Formulation," AIAA Paper 97-0643, Jan. 1997; also ICASE Report No. 97-9 (NASA CR 201650), Jan. 1997.

6. Iollo, A., Salas, M., Ta'asan, S., "Shape Optimization Governed by the Euler Equations Using an Adjoint Method," ICASE Report No. 93-78 (NASA CR 191555), Nov. 1993.

7. Borggaard, J. T., Burns, J., Cliff, E. M., and Gunzburger, M. D., "Sensitivity Calculations for a 2-D Inviscid Supersonic Forebody Problem," Identification and Control of Systems Governed by Partial Differential Equations, SIAM Publications, Philadelphia, 1993, pp. 14-24.

8. Godfrey, A. G., and Cliff, E. M., "Direct Calculation of Aerodynamic Force Derivatives: A Sensitivity-Equation Approach," AIAA Paper 98-0393, Jan. 1998.

9. Bischof, C., and Griewank, A., "ADIFOR: A Fortran System for Portable Automatic Differentiation," AIAA Paper 92-4744 CP, Sept. 1992. 
10. Bischof, C., Carle, A., Corliss, G., Griewank, A., and Hovland, P., "ADIFOR: Generating Derivative Codes from Fortran Programs," Scientific Programming, Vol. 1, No. 1, 1992, pp. 11-29.

11. Bischof, C., Carle, A., Khademi, P., Mauer, A., “ADIFOR 2.0: Automatic Differentiation of Fortran 77 Programs," IEEE Computational Science and Engineering, Vol. 3, No. 3, 1996, pp. 18-32.

12. Oloso, A., and Taylor III, A. C., "Aerodynamic Shape-Sensitivity Analysis and Design Optimization on the IBM-SP2 Using the 3D Euler Equations," AIAA Paper 97-2273, June 1997.

13. Taylor III, A. C., "Automatic Differentiation of Advanced Flow-Analysis Codes in Incremental Iterative Form for Multidisciplinary Applications," Final Report, Master Contract NAS1-19858 (Task No. 77), Nov. 1995; available from Old Dominion University Research Foundation, ODURF Report No. 96-147, Old Dominion University, Norfolk, VA, 1996.

14. Taylor III, A. C., Oloso, A., and Newman III, J. C., "CFL3D.ADII (Version 2.0): An Efficient, Accurate, General-Purpose Code for Flow Shape-Sensitivity Analysis," AIAA Paper 97-2204, June 1997.

15. Green, L. L., Newman, P. A., and Haigler, K. J., "Sensitivity Derivatives for Advanced CFD Algorithms and Viscous Modelling Parameters Via Automatic Differentiation," Journal of Computational Physics, Vol. 125, No. 2, 1996, pp. 313-324; also AIAA Paper 93-3321, July 1993.

16. Carle, A., Green, L. L., Bischof, C. H., and Newman, P. A., "Applications of Automatic Differentiation in CFD," AIAA Paper 94-2197, June 1994.

17. Bischof, C., Corliss, G., Green, L., Griewank, A., Haigler, K., and Newman, P.,"Automatic Differentiation of Advanced CFD Codes for Multidesciplinary Design," Computing Systems in Engineering, Vol. 3, No. 6, 1992, pp. 625-637.

18. Thomas, A., Smith, R. E., and Tiwari, S. N.,"Aerodynamic Shape Optimization of Blended Surfaces Representing HSCT-Type Config ırations," AIAA Paper 95-1826, June 1995.

19. Mohammadi, B., "Optimal Shape Design, Reverse Mode of Automatic Differentiation and Turbulence," AIAA Paper 97-0099, Jan. 1997.

20. Carle, A., Fagan, M., and Green, L. L., "Preliminary Results from the Application of an Automatic Adjoint Code Generator to CFL3D," Center for Research on Parallel Computation, CRPC-TR98741, Rice University, Houston, Feb. 1998.

21. Mohammadi, B., Male, J.-M., and Rostaing-Schmidt, N., "Automatic Differentiation in Direct and Reverse Modes: Application to Optimum Shapes Design in Fluid Mechanics," Computational Differentiation, edited by Berz, M., 3ischof, C., Corliss, G., and Griewank, A., SIAM Proceedings Series, SIAM, Philadelphia, 996, pp. 309-318.

22. Labrujere, T. E., and Slooff, J. W., "Computational Methods for the Aerodynamic Design of Aircraft Components," Annual Review of Fluid Mechanics, Vol. 25, 1993, pp. 183214.

23. Taylor III, A. C., Newman, P. A., Hou, G. J.-W., and Jones, H. E., "Recent Advances in Steady Compressible Aerodynamic Sensitivity Analysis," Flow Control, edited by Gunzburger, M. D., Vol. 68, The IMA Volumes in Mathematics and its Applications, Springer-Verlag, New York, 1995, pp. 341-355.

24. Periaux, J., and Degrez, G. (eds.), Optimum Design Methods in Aerodynamics AGARDFDP-VKI Special Course, von Karman Institute for I luid Dynamics, Rhode Saint Genese, Belgium, April 1994.

25. Van den Braembussche, R. A., and Manna, M. (eds.), Inverse Design and Optimisation Methods, VKI Lecture Series 1997-05, von Karman Institute for Fluid Dynamics, Rhode Saint Genese, Belgium, April 1997.

26. Jameson, A., "Essential Elements of Computational Algorithms for Aerodynamic Analysis and Design," ICASE Report No. 97-68 (NASA CR-97-206268), Dec. 1997.

27. Hicks, R. M., Murman, E. M., and Vanderplaats, G. V., "An Assessment of Airfoil Design by Numerical Optimization," NASA TM-3092, July 1974.

28. Vanderplaats, G. N., and Hicks, R. M., "Numerical Airfoil Optimization Using Reduced Number of Design Coordinates," NASA TM-73151, July 1976. 
29. Hicks, R. M., and Henne, P. A., "Wing Design by Numerical Optimization," AIAA Paper 77-1247, Aug. 1977.

30. Pironneau, O., "On Optimum Profiles in Stokes Flow," Journal of Fluid Mechanics, Vol. 59,1973 , pp. 117-128.

31. Pironneau, O., "On Optimum Design in Fluid Mechanics," Journal of Fluid Mechanics, Vol. 64, 1974, pp. 97-110.

32. Glowinski, R., and Pironneau, O., "On the Numerical Computation of the Minimum-Drag Profile in Laminar Flow," Journal of Fluid Mechanics, Vol. 72, part 2, 1975, pp. 385389.

33. Angrand, F., "Optimal Design for Potential Flows," International Journal for Numerical Methods in Fluids, Vol. 13, 1983, pp. 265-282.

34. Pironneau, O., Optimal Shape Design for Elliptic Systems, Springer Series in Computational Physics, Springer-Verlag, New York, 1984.

35. Sobieszczanski-Sobieski, J., "The Case for Aerodynamic Sensitivity Analysis," Sensitivity Analysis in Engineering, compiled by Adelman, H. M., and Haftka, R. T., NASA CP 2457 , Feb. 1987, pp. 77-96.

36. Elliot, J., and Peraire, J., "Aerodynamic Design and Using Unstructured Meshes," AIAA Paper 96-1941, June 1996.

37. Eleshaky, M. E., "A Computational Aerodynamic Design Optimization Method Using Sensitivity Analysis," Ph.D. Dissertation, Old Dominion University, Norfolk, VA, May 1992.

38. Burgreen, G. W., "Three-Dimensional Aerodynamic Shape Optimization Using Discrete Sensitivity Analysis," Ph.D. Dissertation, Old Dominion University, Norfolk, VA, May 1994.

39. Joh, C.-Y., Grossman, B., and Haftka, R. T., "Efficient Optimization Procedure for Transonic Airfoil Design," ASME Winter Annual Meeting, Computational Structural Mechanics and Multidisciplinary Optimization, Book No. H00534, Dec. 1989, pp. 67-76.

40. Malone, J. B., Narramore, J. C., and Sankar, L. N., "An Efficient Airfoil Design Method Using the Navier-Stokes Equations," Computational Methods for Aerodynamic Design (Inverse) and Optimization, AGARD CP-463, March 1990, pp. 5.1-5.18.

41. Newman III, J. C., "Integrated Multidisciplinary Design Optimization Using Discrete Sensitivity Analysis for Geometrically Complex Aeroelastic Configurations," Ph.D. Dissertation, Virginia Polytechnic Institute and State University, Blacksburg, VA, July 1997.

42. Elliot, J., and Peraire, J., "Practical 3D Aerodynamic Design and Optimization Using Unstructured Grids," AIAA Paper 96-4170 CP, Sept. 1996.

43. Korivi, V. M., Taylor III, A. C., Newman, P. A., Hou, G. J.-W., and Jones, H. E., “An Approximate Factored Incremental Strategy for Calculating Consistent Discrete CFD Sensitivity Derivatives," Journal of Computational Physics, Vol. 113, 1994, pp. 336-346; also NASA TM 104207, Feb. 1992.

44. Huffman, W. P., Melvin, R. G., Young, D. P., Johnson, F. T., Bussoletti, J. E., Bieterman, M. B., and Hilmes, C. L., "Practical Design and Optimization in Computational Fluid Dynamics," AIAA Paper 93-3111, July 1993.

45. Bischof, C. H., Green, L. L., Haigler, K. J., and Knauff, T. L., "Parallel Calculation of Sensitivity Derivatives for Aircraft Design Using Automatic Differentiation," AIAA Paper 94-4261 CP, Sept. 1994.

46. Felker, F. F., "Calculation of Optimum Airfoils Using Direct Solutions of the NavierStokes Equations," AIAA Paper 93-3323, July 1993.

47. Huddleston, D. H., Soni, B. K., and Zheng, X., "Application of a Factored NewtonRelaxation Scheme to Calculation of Discrete Aerodynamic Sensitivity Derivatives," AIAA Paper 94-1894, June 1994.

48. Rizk, M. H., "Application of the Single-Cycle Optimization Approach to Aerodynamic Design," Journal of Aircraft, Vol. 22, No. 6, 1985, pp. 509-515.

49. Kuruvila, G., Ta'asan, S., and Salas, M. D., "Airfoil Optimizations by the One-Shot Method," Optimum Design Methods in Aerodynamics, AGARD-FDP-VKI Special Course, von Karman Institute for Fluid Dynamics, Rhode Saint Genese, Belgium, April 1994.

50. Campbell, R. L., "An Approach to Constrained Aerodynamic Design with Application to Airfoils," NASA TP-3260, Nov. 1992. 
51. Orozco, C., and Ghattas, O., "Optimum Design of Systems Governed by Nonlinear Partial Differential Equations," AIAA Paper 92-4836 CP, Sept. 1992.

52. Korivi, V. M., "Aerodynamic Design Optimization with Consistently Discrete Sensitivity Derivatives via the Incremental Iterative Method," Fh.D. Dissertation, Old Dominion University, Norfolk, VA, May 1995.

53. Horibata, Y., "Airfoil Design Using an Optimization Procedure and the Implicit Function Theorem," AIAA Paper 97-0513, Jan. 1997.

54. Chattopadhyay, A., and Pagaldipti, N., "A Multilevel Decomposition Procedure for Efficient Design of High Speed Civil Transport," AIAA Paper 94-0097, Jan. 1994.

55. Korivi, V. M., Newman, P. A., and Taylor III, A. C. "Aerodynamic Optimization Studies Using a 3-D Supersonic Euler Code with Efficient Calculation of Sensitivity Derivatives," AIAA Paper 94-4270 CP, Sept. 1994.

56. Soemarwoto, B. I., "Multi-Point Aerodynamic Design by Optimization," Ph. D. Dissertation, Delft University of Technology, Delft, the Netherlands, Dec. 1996.

57 Newman III, J. C., and Taylor III, A. C., "Three-Dimensional Aerodynamic Shape Sensitivity Analysis and Design Optimization Using the Euler Equations on Unstructured Grids," AIAA Paper 96-2464, June 1996.

58. Newman III, J. C., Taylor III, A. C., and Barnwell, R. W., "Aerodynamic Shape Sensitivity Analysis and Design Optimization of Complex Configurations Using Unstructured Grids,", AIAA Paper 97-2275, June 1997.

59. Barth, T. J., and Linton, S. W., "An Unstructured Mesh Newton Solver for Compressible Fluid Flow and Its Parallel Implementation," AIAA Paper 95-0221, Jan. 1995.

60. Brown, P. N., and Saad, Y., "Hybrid Krylov Methods for Nonlinear Systems of Equations," SIAM Journal on Scientific and Statistical Computing, Vol. 11, No. 3, 1990 , pp. 450-481.

61. Hou, G. J.-W., Korivi, V. M., Taylor III, A. C., Maroju, V., and Newman, P. A., "Simultaneous Aerodynamic Analysis and Design (ptimization (SAADO) of Turbulent Transonic Airfoil Using a Navier-Stokes Code with Automatic Differentiation (ADIFOR)," Computational Aerosciences Workshop 95, edited by Feiereisen, W. J., and Lacer, A. K., NASA CD CP 20010, Jan. 1996, pp. 82-85.

62. Haftka, R. T., "Simultaneous Analysis and Design," AIAA Journal, Vol. 23, No. 7, 1985, pp. 1099-1103.

63. Rizk, M. H., "Optimization by Updating Design Parameters as CFD Iterative Flow Solutions Evolve," Multidisciplinary Applications of CFD, edited by Baysal, O., ASMEFED, Vol. 129, Winter Annual Meeting, Dec. 1991 pp.51-62.

64. Iollo, A., Kuruvila, G., and Ta'asan, S., "Psuedo-Tirne Method for Optimal Shape Design Using the Euler Equations," ICASE Report No. 95-59 (NASA CR 198205), Aug. 1995.

65. Ta'asan, S., "Pseudo-Time Methods for Constrained Optimization Problems Governed by PDE," ICASE Report No. 95-32 (NASA CR 195081), May 1995.

66. Reuther, J., Jameson, A., Alonso, J. J., Rimlinger, M J., and Saunders, D., "Constrained Multipoint Aerodynamic Optimization Using an Adjoint Formulation and Parallel Computers," AIAA Paper 97-0103, Jan. 1997.

67. Reuther, J., Jameson, A., Farmer, J., Martinelli, L., a 1d Saunders, D., “Aerodynamic Shape Optimization of Complex Aircraft Configurations v: a an Adjoint Formulation," AIAA Paper 96-0094, Jan. 1996.

68. Arabshahi, S., and Whitfield, D. L., "A Multiblock Approach to the Three-Dimensional Unsteady Euler Equations About a Wing-Pylon-Sto e Configuration," AIAA Paper 893401 , Aug. 1989.

69. Ghaffari, F., Luckring, J. M., Thomas, J. L., Bates, B. L., and Biedron, R. T., "Multiblock Navier-Stokes Solutions About the F/A-18 Wing-LFX-Fuselage Configuration," Journal of Aircraft, Vol. 30, No. 3, 1993, pp. 293-303.

70. Walters, R. W., Reu, T., McGrory, W. D., Thomas, J. L., Richardson, P. F., "A Longitudinally-Patched Grid Approach with Applicstions to High Speed Flows," AIAA Paper 88-0715, Jan. 1988.

71. Thomas, J. L., Walters, R. W., Reu, T., Ghaffari, F., Weston, R. P., and Luckring, J. M., “A Patched-Grid Algorithm for Complex Configuration; Directed Toward the F/A-18 Aircraft," AIAA Paper 89-0121, Jan. 1989. 
72. Meakin, R. L., "Moving Body Overset Grid Methods for Complete Aircraft Tiltrotor Simulations," AIAA Paper 93-3350, July 1993.

73. Baysal, O., Fouladi, K., and Lessard, V. R., "Multigrid and Upwind Viscous Flow Solver on 3-D Overlapped and Embedded Grids," AIAA Journal, Vol. 29, No. 4, 1991, pp. $903-$ 910 .

74. Steger, J. L., Dougherty, F. C., and Benek, J. A., "A Chimera Grid Scheme," Advances in Grid Generation, ASME-FED,Vol. 5, June 1983, pp. 59-69.

75. Slack, D. C., Walters, R. W., and Lohner, R., "An Interactive Adaptive Remeshing Algorithm for the Two-Dimensional Euler Equations," AIAA Paper 90-0331, Jan. 1990.

76. Rausch, R. D., Batina, J. T., and Yang, H. T. Y., "Spatial Adaptation Procedures on Unstructured Meshes for Accurate Unsteady Aerodynamic Flow Computations," AIAA Paper 91-1106, April 1991.

77. Venkatakrishnan, V., "Perspective on Unstructured Grid Flow Solvers," AIAA Journal, Vol. 34, No. 3, 1996, pp. 533-547; also AIAA Paper 95-0667, Jan. 1995 or ICASE Report No. 95-3 (NASA CR 195025), Feb. 1995.

78. Jameson, A., Pierce, N. A., and Martinelli, L., "Optimum Aerodynamic Design Using the Navier-Stokes Equations," AIAA Paper 97-0101, Jan. 1997.

79. Eleshaky, M. E., and Baysal, O., "Shape Optimization of a 3D Nacelle Near a Flat Plate Wing Using Multiblock Sensitivity Analysis," AIAA Paper 94-0160, Jan. 1994.

80. Lacasse, J. M!, and Baysal, O., "Shape Optimization of Single- and Two-Element Airfoils on Multiblock Grids," AIAA Paper 94-4273 CP, Sept. 1994.

81. Thomas, J. L., Krist; S. T., and Anderson, W. K., "Navier-Stokes Computations of Vortical Flows Over Low Aspect Ratio Wings," AIAA Journal, Vol. 28, No. 2, 1990, pp. 205-212.

82. Newman, P. A., Hou, G. J.-W., and Taylor III, A. C., "Observations Regarding Use of Advanced CFD Analysis, Sensitivity Analysis, and Design Codes in MDO,"

Multidisciplinary Design Optimization: State of the Art, edited by Alexandrov, N. M. and Hussaini, M. Y., SIAM Proceedings Series, SIAM, Philadelphia, 1997, pp. 263-279; also ICASE Report 96-16 (NASA CR 198293), Mar. 1996.

83. Sherman, L. L., Taylor III, A. C., Green, L. L., Newman, P. A., Hou, G. J.-W., and Korivi, V. M., "First- and Second-Order Aerodynamic Sensitivity Derivatives via Automatic Differentiation with Incremental Iterative Methods," Journal of Computational Physics, Vol. 129, 1996, pp. 307-336; also AIAA Paper 94-4262 CP, Sept. 1994.

84. Baldwin, B., and Lomax, H., "Thin Layer Approximation and Algebraic Model for Separated Turbulent Flows," AIAA Paper 78-0257, Jan. 1978.

85. Hou, G. J.-W., Maroju, V., Taylor III, A. C., Korivi, V. M., and Newman, P. A., "Transonic Turbulent Airfoil Design Optimization Using Automatic Differentiation in Incremental Iterative Form," AIAA Paper 95-1692, June 1995.

86. Vatsa, V. N., and Wedan, B. W., "Development of a Multigrid Code for 3-D NavierStokes Equations and Its Application to a Grid-Refinement Study," Journal of Computers and Fluids, Vol. 18, No. 4, 1990, pp. 391-403.

87. Beux, F., and Dervieux, A., "A Hierarchical Approach for Shape Optimization," INRIA Report 1868, Feb. 1993.

88. Ghattas, O., and Bark, J.-H., "Optimal Control of Two- and Three-Dimensional NavierStokes Flows," Journal of Computational Physics, Vol. 136, 1997, pp. 231-244.

89. Newman III, J. C., Taylor III, A. C., and Burgreen, G. W., "An Unstructured Grid Approach to Sensitivity Analysis and Shape Optimization Using the Euler Equations," AIAA Paper 95-1646, June 1995.

90. Elliot, J., and Peraire, J., "Aerodynamic Optimization on Unstructured Meshes with Viscous Effects," AIAA Paper 97-1849, July 1997.

91. Shrinivas, G. N., "Three-Dimensional Design Methods for Turbomachinery Applications," Ph D Thesis, Oxford University Computing Laboratory, Oxford, 1996.

92. Burgreen, G. W., and Antaki, J. F., "CFD-Based Design Optimization of a ThreeDimensional Rotary Blood Pump," AIAA Paper 96-4185 CP, Sept. 1996.

93. Burgreen, G. W., Antaki, J. F., and Griffith, B. P., "A Design Improvement Strategy for Axial Blood Pumps Using Computational Fluid Dynamics," American Society for Artificial Internal Organs Journal, Vol. 42, 1996, pp. 354-369.

94. Anderson, W. K., and Bonhaus, D. L., "Aerodynamic Design on Unstructured Grids for Turbulent Flows," NASA TM 112867, June 1997. 
95. Spalart, P. R., and Allmaras, S. R., “A One Equation Turbulence Model for Aerodynamic Flows," AIAA Paper 92-0439, Jan. 1992.

96. Mortenson, M. E., Geometric Modeling, John Wiley and Sons, New York, 1985.

97. AIAA White Paper, AIAA Technical Committee on Multidisciplinary Design Optimization (MDO), Jan. 15, 1991.

98. Grose, D. L., "Re-Engineering the Aircraft Design Process," AIAA Paper 94-4323 CP, Sept. 1994.

99. Jameson, A., "Re-Engineering the Design Process Through Computation," AIAA Paper 97-0641, Jan. 1997.

100.Sobieszczanski-Sobieski, J., "Sensitivity Analysis and Multidusciplinary Optimization for Aircraft Design: Recent Advances and Results," Journal of Aircraft, Vol. 27, No. 12, 1990, pp. 993-1001.

101.Sobieszczanski-Sobieski, J., "Multidisciplinary Design and Optimization," Integrated Design Analysis and Optimization of Aircraft Structures, AGARD LS-186, May 1992, pp. 4-1 to 4-15.

102.Sobieszczanski-Sobieski, J., and Haftka, R. T., "Multidisciplinary Aerospace Design Optimization: Survey of Recent Developments," AIAA Paper 96-0711, Jan. 1996.

103.Cramer, E. J., Dennis, J. E., Frank, P. D., Lewis, R. M., and Shubin, G. R., "Problem Formulation for Multidisciplinary Optimization," SIAM Journal of Optimization, Vol. 4, No. 4, 1994, pp. 754-776.

104.Balling, R. J., and Sobieszczanski-Sobieski, J., "Optimization of Coupled Systems: A Critical Overview of Approaches," AIAA 94-4330 CP, Sept. 1994.

105. Newman, P. A., Hou, G. W., Jones, H. E., Taylor III. A. C., and Korivi, V. M., "Observations on Computational Methodologies for Use in Large-Scale, Gradient-Based, Multidisciplinary Design," AIAA Paper 92-4753 CP, Sept. 1992.

106. Newman, P. A., "Preparation of Advanced CFD Codes for Use in Sensitivity Analyses and Multidisciplinary Design Optimization," Optimal Design and Control, edited by Borggaard, Burkardt, Gunzberger, and Peterson, , B irkhauser, Boston, 1995, pp. 241-274.

107. Newman III, J. C., Newman, P. A., Taylor III, A. C., and Hou, G. J-.W., "Efficient Nonlinear Static Aeroelastic Wing Analysis," Submitted to Journal of Computers and Fluids.

108. Arslan, A. E., and Carlson, L. A., "Integrated Determination of Sensitivity Derivatives for an Aeroelastic Transonic Wing," AIAA Paper 94-4400 CP, Sept. 1994.

109. Sobieszczanski-Sobieski, J., "Sensitivity of Complex, Internally Coupled Systems," AIAA Journal, Vol. 28, No. 1, 1990, pp. 153-160; also AIAA Paper 88-2378, April 1988.

110. Barthelemy, J.-F. M., and Bergen, F. D., "Shape Seinsitivity Analysis of Wing Static Aeroelastic Characteristics," NASA TP-2808, May 1988.

111. Newman III, J. C., Newman, P. A., Taylor III, A. C, and Hou, G. J-.W., "Nonlinear Aerodynamic Design Optimization of a Flexible Wiig," AIAA Paper 96-4108 CP, Sept. 1996. 


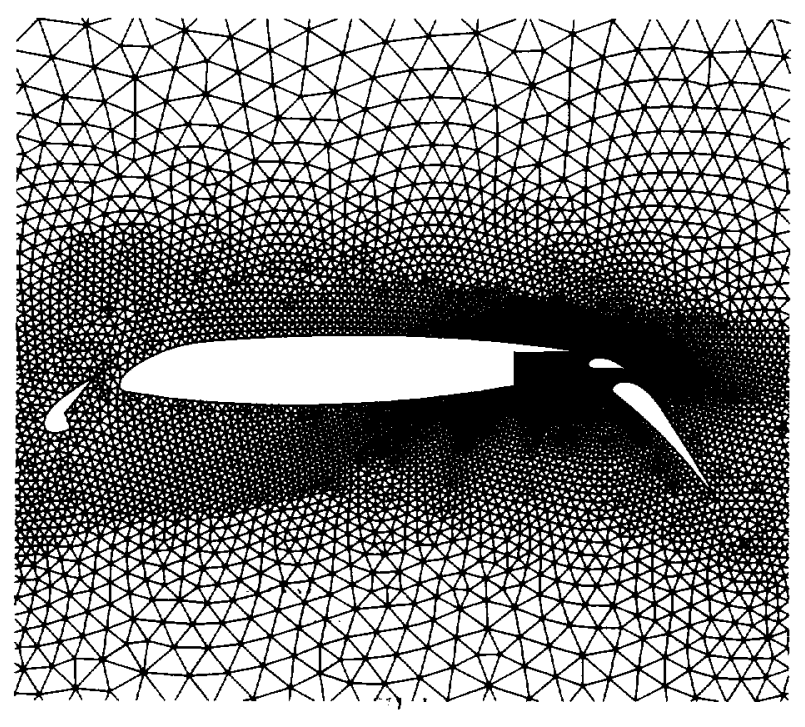

(a) Unstructured Euler mesh (7614 nodes, 14919 cells).

\section{$\because n i s$}

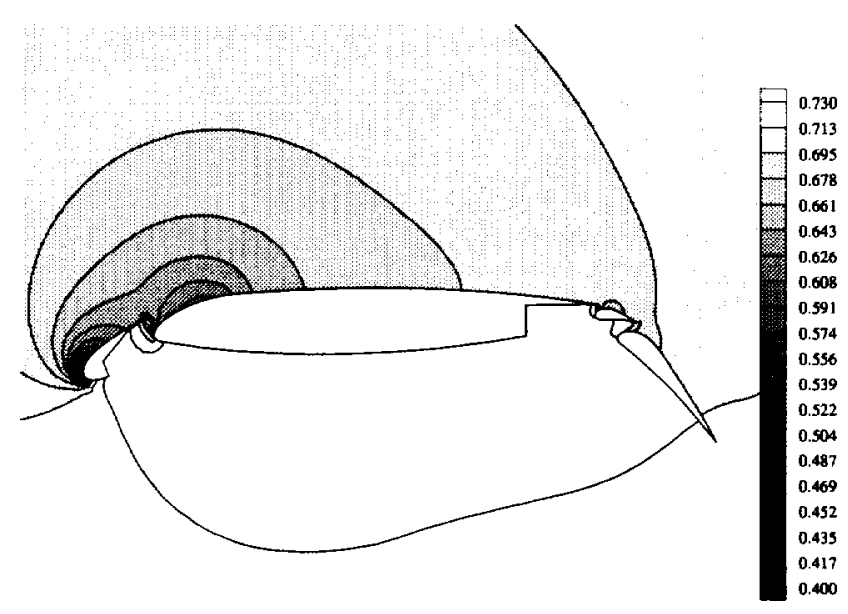

(c) Contours of nondimensional pressure.

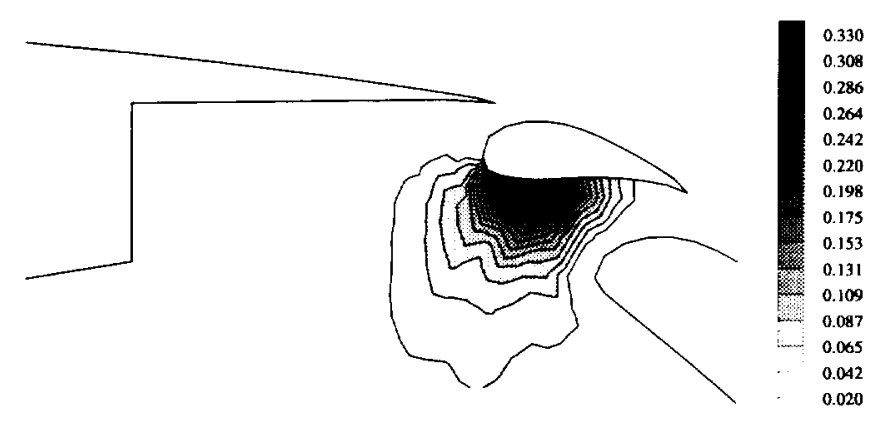

(b) Contours of grid sensitivity; $y$-coordinate with respect to a vane design variable.

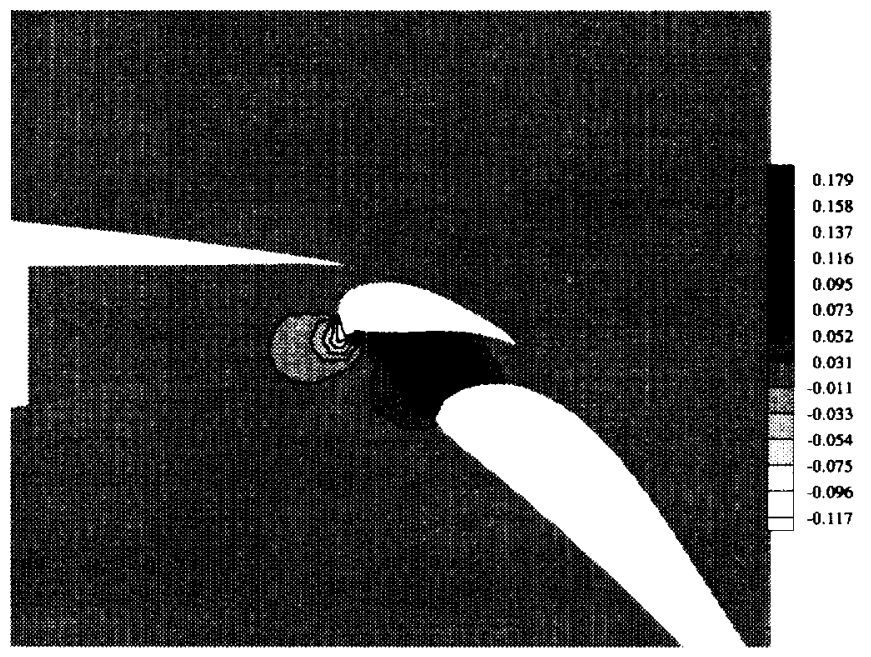

(d) Contours of nondimensional pressure sensitivity with respect to a vane design variable.

Figure 1. Discrete-direct aerodynamic shape sensitivity analysis for a four-element airfoil configuration $\left(M_{\infty}=0.2, \alpha=16.02^{\circ}\right)$. 
(a) Unstructured Euler mesh (7974 nodes, 15630 cells).
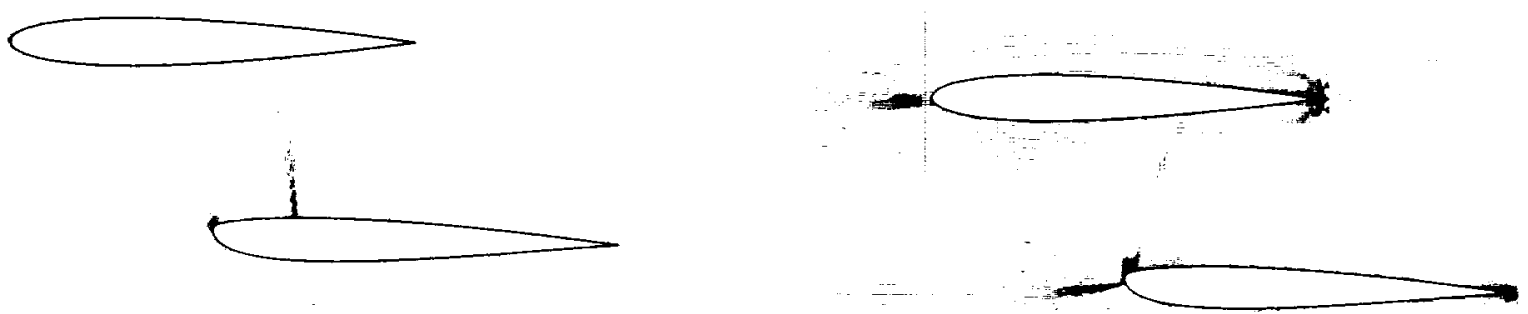

(b) Contours of nondimensional pressure.

(c) C'ontours of costate variable corresponding to st eamwise momentum.

Figure 2. Continuous-adjoint aerodynamic shape sensitivity analysis for a two-element airfoil configuration $\left(M_{\infty}=0.6, \alpha=0^{\circ}\right)$. 


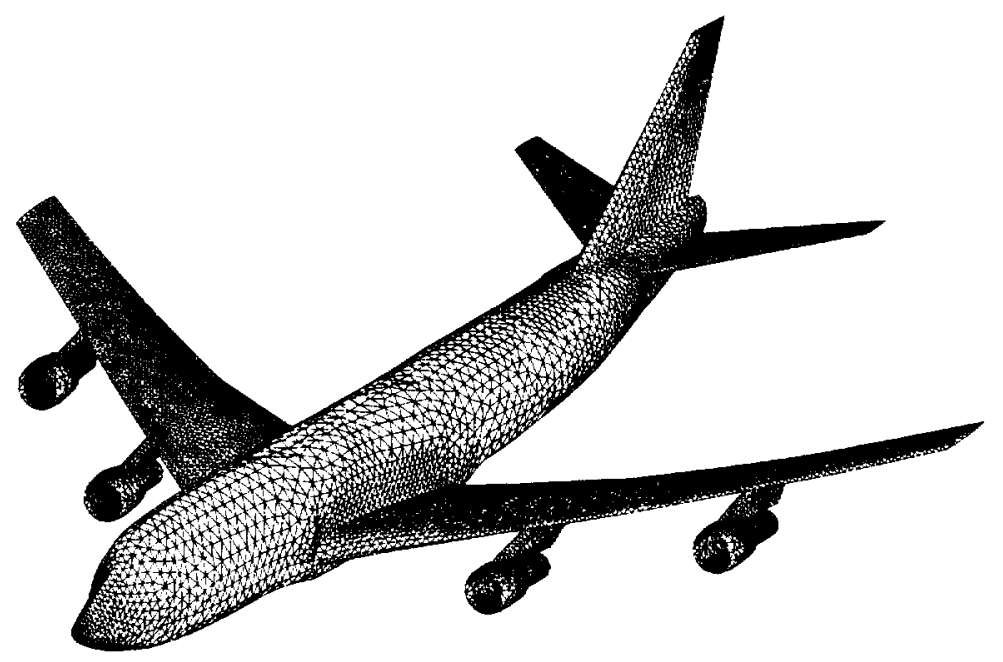

(a) CFD surface grid; unstructured Euler mesh has 63828 nodes and 352547 cells.

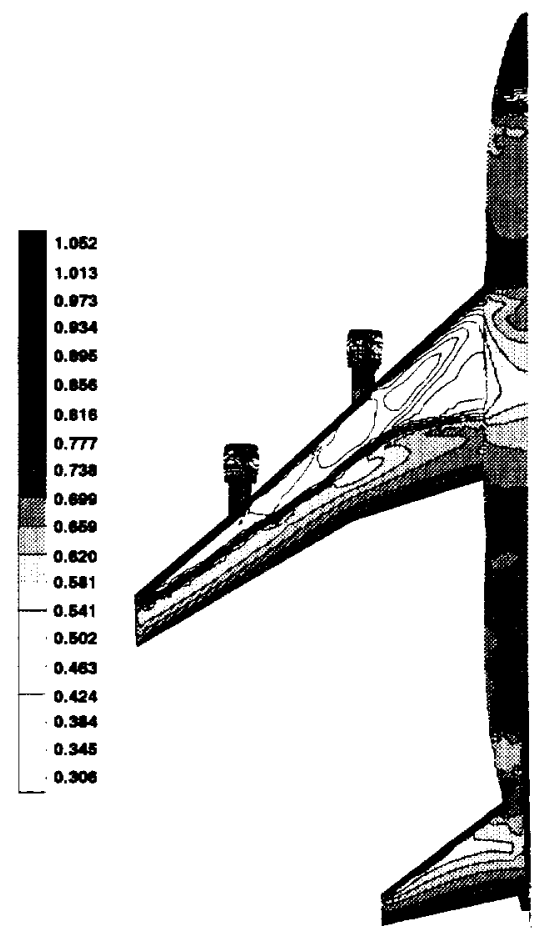

(b) Contours of nondimensional pressure.
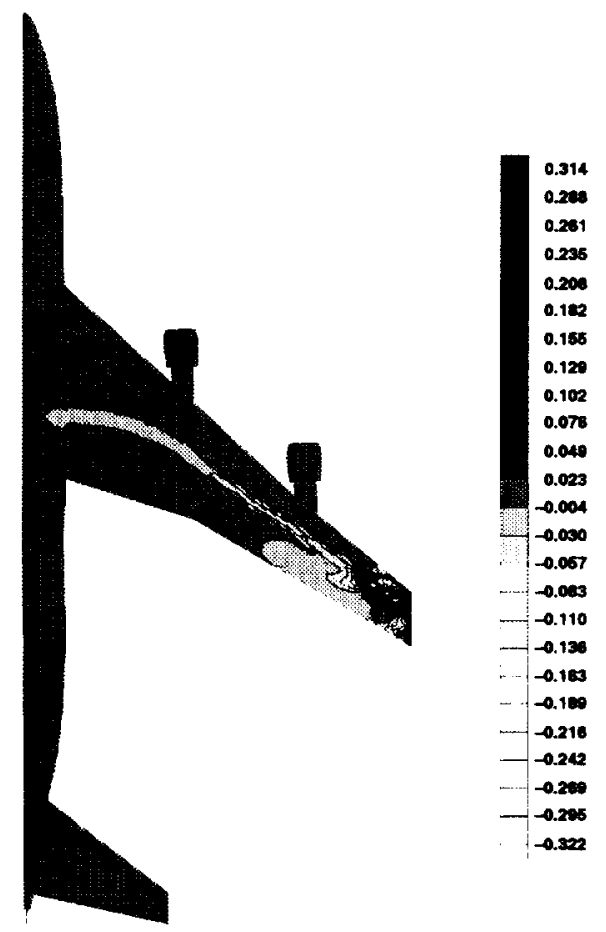

(c) Contours of nondimensional pressure sensitivity with respect to a winglet-type design variable.

Figure 3. Discrete-direct aerodynamic shape sensitivity analysis for an aircraft configuration approximating a Boeing $747-200\left(M_{\infty}=0.84, \alpha=2.73^{\circ}\right)$. 


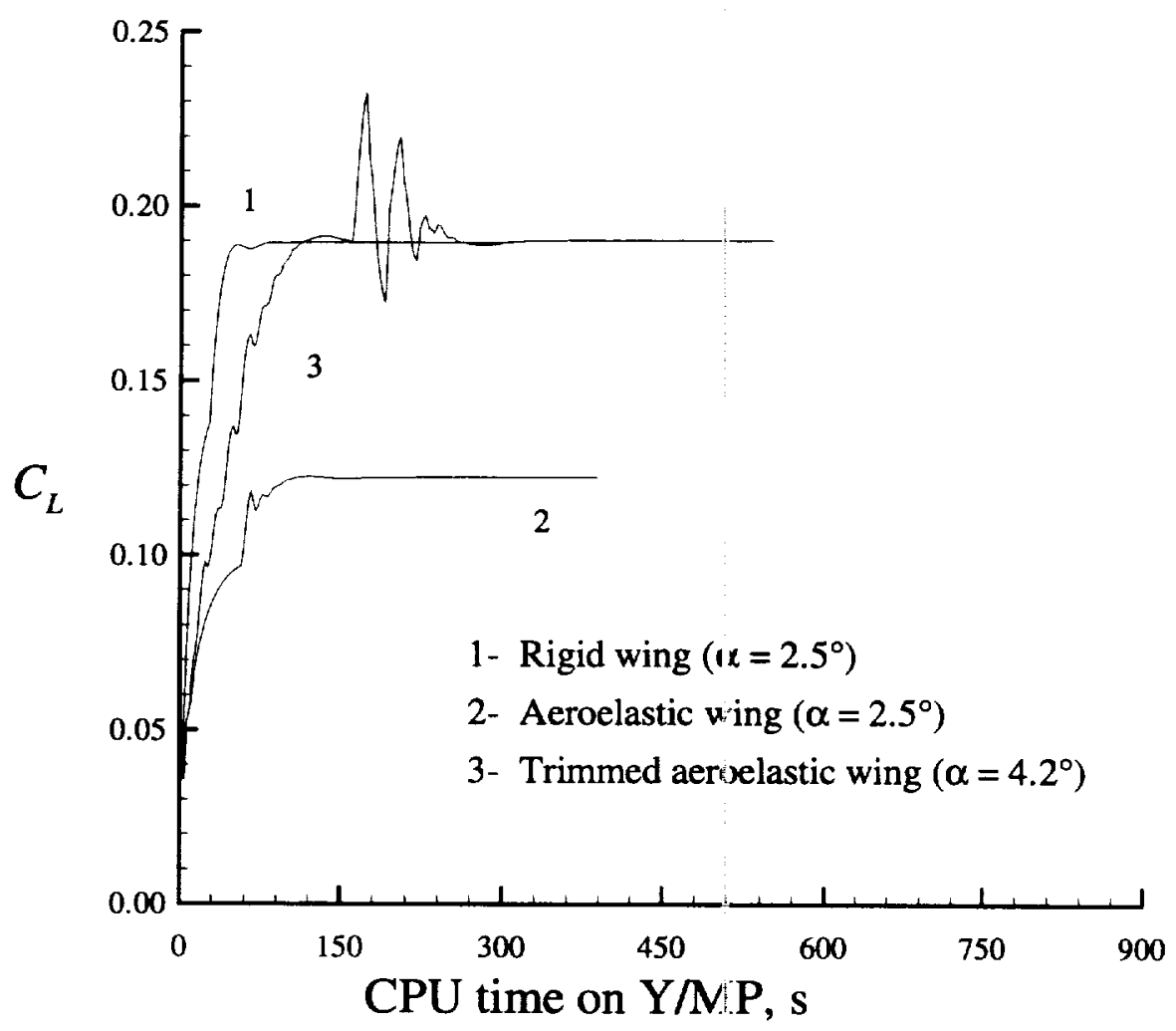

(a) Lift coefficient convergence histories.

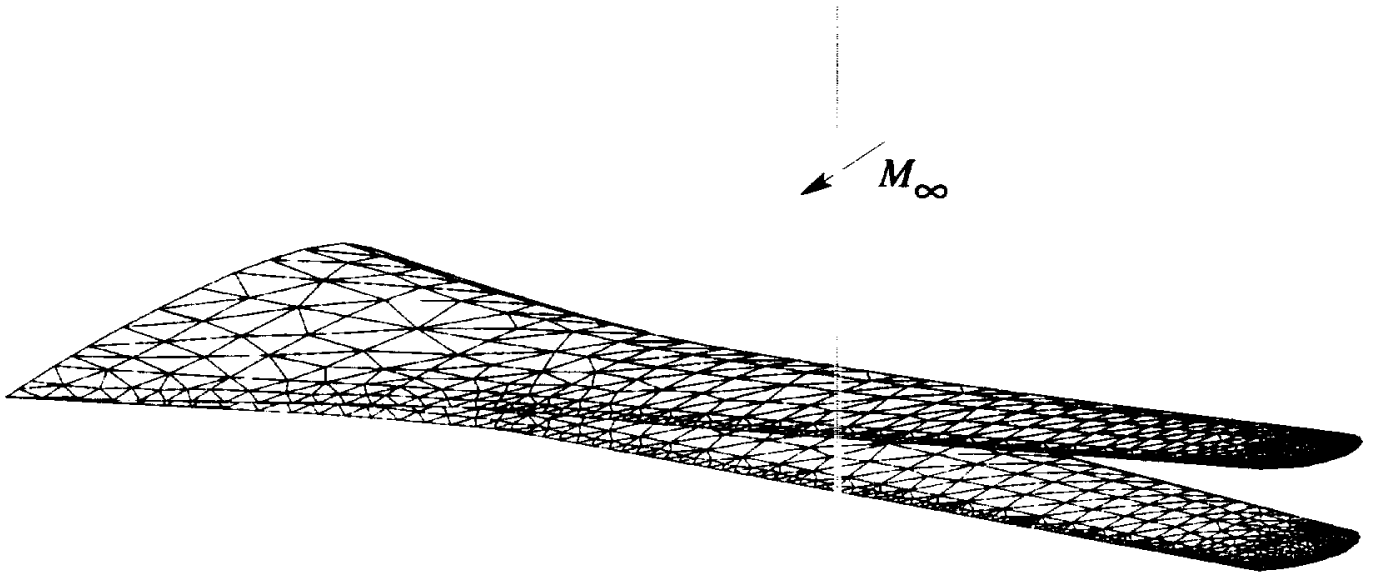

(b) CFD surface grids for initial rigid wing an 1 deflected aeroelastic wing.

Figure 4. Aerodynamic and static aeroelastic analysis for an aircraft wing $\left(M_{\infty}=0.85, \alpha=2.5^{\circ}\right.$, alt. $=35000 \mathrm{ft}$ ). Unstructured Euler mesh has 3867 norles and 18856 cells; structural finiteelement analysis has 159 degrees of freedom. 